Grand Valley State University

ScholarWorks@GVSU

$9-2004$

\title{
Legibility, Mystery, and Visual Access as Predictors of Preference and Perceived Danger in Forest Settings without Pathways
}

Thomas R. Herzog

Grand Valley State University, herzogt@gvsu.edu

Laura S. Kropscott

Grand Valley State University

Follow this and additional works at: https://scholarworks.gvsu.edu/psy_articles

Part of the Psychology Commons

\section{ScholarWorks Citation}

Herzog, Thomas R. and Kropscott, Laura S., "Legibility, Mystery, and Visual Access as Predictors of Preference and Perceived Danger in Forest Settings without Pathways" (2004). Peer Reviewed Articles. 32.

https://scholarworks.gvsu.edu/psy_articles/32

This Article is brought to you for free and open access by the Psychology Department at ScholarWorks@GVSU. It has been accepted for inclusion in Peer Reviewed Articles by an authorized administrator of ScholarWorks@GVSU. For more information, please contact scholarworks@gvsu.edu. 


\section{LEGIBILITY, MYSTERY, AND VISUAL ACCESS AS PREDICTORS OF PREFERENCE AND PERCEIVED DANGER IN FOREST SETTINGS WITHOUT PATHWAYS}

THOMAS R. HERZOG is a professor of psychology at Grand Valley State University in Allendale, Michigan. His current research focuses on environmental preferences, restorative environments, and the psychology of humor.

LAURA S. KROPSCOTT received her bachelor's degree in psychology from Grand Valley State University.

ABSTRACT: The authors attempt to address some unresolved issues within the Kaplans' preference-matrix model of environmental preference. These issues involve the relation between legibility and preference and the relations among preference, danger, and mystery. Participants rated 70 within-forest settings containing no visible pathways for preference, danger, or one of seven predictor variables. Legibility and coherence were found to be independent, positive predictors of preference. Likewise, landmarks and visual access were independent positive predictors of legibility. Legibility fully mediated the positive relation between landmarks and preference. Visual access interacted with legibility in predicting preference: The relation between preference and either predictor was strongest and positive at low values of the other predictor. Preference and danger were negatively correlated, and there was some tendency for each of them to have a different pattern of relations with predictors. Mystery was negatively correlated with both preference and visual access, an unusual finding.

Keywords: legibility; mystery; access; preference; danger

The study reported in this article represents an integration of ideas from two related lines of research. The first line of research was an attempt to discover why legibility, one of the theoretical predictors in the Kaplans' preference matrix (R. Kaplan \& Kaplan, 1989; S. Kaplan \& Kaplan, 1982), had

ENVIRONMENT AND BEHAVIOR, Vol. 36 No. 5, September 2004 659-677

DOI: $10.1177 / 0013916504264138$

(c) 2004 Sage Publications 
such a lackluster empirical record as a predictor of environmental preference. The second line of research dealt with the intriguing fact that preference and perceived danger are not simply polar opposites, although they tend to be negatively correlated. Instead, preference and danger tend to have somewhat different patterns of relations with predictor variables. In particular, one of the Kaplans' predictor variables, mystery, tends to be positively related to both preference and danger in certain contexts, a pattern described as paradoxical by Herzog and Miller (1998). Below, we examine how each of these lines of research contributed to the present study. Given that the Kaplans' approach to environmental preference has had substantial theoretical and practical influence, any exploration of unresolved issues within their framework would seem to be a useful contribution.

Regarding the first line of research, Herzog and Leverich (2003) were struck by the uneven track records of the theoretical predictors in the Kaplans' preference matrix. For those readers in need of a refresher, the preference matrix is composed of two binary dimensions. One deals with the basic human needs of understanding and exploration (known in earlier writings as making sense and involvement). The other deals with whether one is processing the two-dimensional picture plane, where the information is immediately available, or the larger three-dimensional world, which requires greater inference on the part of the perceiver. Together, these two dimensions define four cells, each of which contains a conceptually distinct predictor of environmental preference. Coherence refers to features of the picture plane that aid in organizing or understanding the scene. Legibility refers to features of the larger environment that foster understanding by aiding way finding and the building of a useful cognitive map. Complexity refers to how much is going on in the two-dimensional scene-how intricate or visually rich it is. Mystery refers to any features that encourage one to enter more deeply into the larger environment with the promise that one could gain interesting new information. Coherence and legibility satisfy the basic need for understanding, whereas complexity and mystery provide opportunities for exploration. In general, the Kaplans propose that all four predictors will be positively related to preference.

Herzog and Leverich (2003) noted that legibility, the last of the preferencematrix predictors to be proposed, also had the weakest track record as a preference predictor. They also noted that in studies including coherence and legibility, the two predictors tended to be strongly positively correlated, and

AUTHORS' NOTE: Correspondence concerning this article should be addressed to Thomas R. Herzog, Department of Psychology, Grand Valley State University, Allendale,Mi 49401; e-mail: herzogt@gvsu.edu. 
coherence tended to be the dominant predictor. Herzog and Leverich tried to separate the two predictors empirically by a purposive selection of field or forest settings representing all combinations of high and low values of each predictor and by the use of alternative definitions of the predictors. In general, these maneuvers proved unsuccessful. However, when they examined their field and forest settings separately, the researchers discovered that legibility and coherence both had significant positive partial relations with preference for the forest settings. They speculated that visual access may be an important component of legibility in within-forest settings and showed in post hoc analyses that both rated openness and rated presence of landmarks were significant positive predictors of legibility for such settings.

Given that the most intriguing findings and theoretical suggestions of Herzog and Leverich (2003) were essentially post hoc, one major purpose of the present study was to examine their proposals in a study specifically designed to do so. Hence, the present study included a much larger sample (70 vs. 21) of within-forest settings. In addition, because the two proposed components of legibility for forest settings, visual access and the presence of landmarks, tend to be confounded when pathways are present, we excluded pathway settings from our sample. Visual access varied primarily as a function of depth of view in our forest settings without pathways, and landmarks varied independently (we hoped) as a function of the presence of distinctive features such as rocks or unusually shaped trees. Given these conditions and the theoretical speculations of Herzog and Leverich, we were prepared to state the following a priori hypotheses:

Hypothesis 1: Legibility and coherence will be independent positive predictors of preference.

Hypothesis 2: Visual access and landmarks will be independent positive predictors of legibility.

Furthermore, given the causal pathway implied by the first two hypotheses, we also thought it useful to examine a proposal about mediation:

Hypothesis 3: The effects of either visual access or landmarks on preference will be mediated by the effect of legibility on preference.

Assessing Hypothesis 3 requires mediation analysis (Baron \& Kenny, 1986; Evans \& Lepore, 1997). We must show that a proposed independent variable (visual access or landmarks) is correlated with both the proposed mediator (legibility) and the dependent variable (preference), that the mediator is correlated with the dependent variable, and that the independent and 


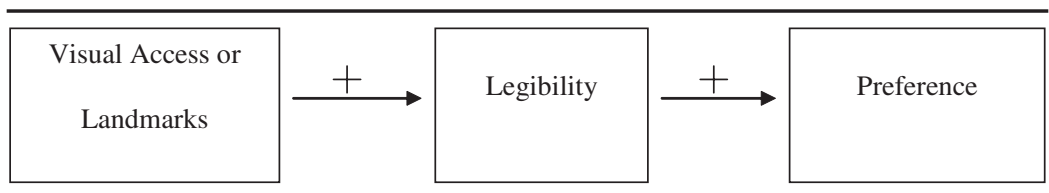

Figure 1: Proposed Mediation Model Involving Either Visual Access or Landmarks as the Independent Variable, Legibility as a Mediating Variable, and Preference as the Dependent Variable

NOTE: Full mediation is depicted, and all relations are positive.

mediator variables do not interact. ${ }^{1}$ If these conditions are met, then a multiple regression analysis is carried out with both the independent variable and the potential mediator included as predictors. Mediation is indicated if the mediator is a significant predictor in the regression analysis and the predictive power of the independent variable is either substantially reduced (partial mediation) or eliminated (full mediation). The full mediation version of Hypothesis 3 is illustrated in Figure 1.

The second line of research that influenced the present study includes studies that have examined the relation between environmental preference and perceived danger as well as the pattern of their relations with predictor variables. There seems to be widespread agreement that preference and danger should be negatively related (e.g., Herzog \& Miller, 1998; Nasar \& Jones, 1997). Several studies have provided empirical confirmation for a variety of setting domains (Herzog \& Flynn-Smith, 2001; Herzog \& Kutzli, 2002; Herzog \& Miller, 1998; Herzog \& Smith, 1988). Moreover, most of the recent research suggests that the negative correlation is not so great as to justify the conclusion that the two variables are simply polar opposites. In the Herzog studies cited above, the correlation between preference and danger within well-defined setting domains (e.g., urban alleys or field or forest settings), where available, has ranged from -.26 to -.75 . Therefore, we propose the following:

Hypothesis 4: The correlation between preference and danger will be negative but not excessive in magnitude.

The clear implication of recent research on preference and danger is that they may be sufficiently independent constructs that the pattern of their relations with common predictor variables will differ at least somewhat from a polar-opposite pattern. Thus, although the studies cited above do generally show that predictors tend to have opposite-sign correlations with preference and danger, there is an intriguing exception. Mystery tends to be positively related to both preference and danger, a result that tends to manifest most 
clearly after controlling for other predictors via regression analysis (Herzog \& Flynn-Smith, 2001; Herzog \& Kutzli, 2002; Herzog \& Miller, 1998). Because this implies that mystery might contribute to either a positive outcome (preference) or to a negative outcome (danger, fear), there has been lively speculation about how to use mystery in such a way as to achieve the former and avoid the latter (e.g., Herzog \& Miller, 1998; Kuo, Bacaicoa, \& Sullivan, 1998; Nasar \& Jones, 1997). For present purposes, however, the key issue is this: Which other predictors should be controlled in examining the relation between mystery and either preference or danger? The initial studies on this issue (Herzog \& Flynn-Smith, 2001; Herzog \& Miller, 1998) simply controlled all other predictors and did not isolate the ones that might be most relevant. However, Herzog \& Kutzli (2002) controlled each of the other predictor in turn and found that predictors related to visibility (especially visual access and visual penetration) had the strongest impact. When these predictors were controlled, the correlation between mystery and either preference or danger was positive and significant. Thus, we have the following hypotheses:

Hypothesis $5 a$ : The pattern of relations for preference and danger with common predictors will differ from a polar-opposite pattern.

Hypothesis 5b: After controlling for visual access, mystery will have a positive relation with both preference and danger.

Finally, we must inquire about the simple correlation between mystery and preference. Anyone possessing even a nodding acquaintance with the environmental preference literature (e.g., R. Kaplan \& Kaplan, 1989) would think this foolish because the answer is obvious. The correlation is positive: Mystery enhances preference. Given this background, Herzog and Kutzli (2002) were discomfited to find a mystery-preference correlation of -.17 in their study of field or forest settings. Although the correlation was not significant, it was sufficiently disturbing to the researchers that they undertook further analyses aimed at reconciliation with previous research. These were the analyses noted above in which they partialed out each of the other predictor variables in turn from the mystery-preference relation and discovered that partialing out predictors related to visibility turned the mystery-preference correlation positive. We must ask the following: Will something similar happen when the sample of settings consists solely of within-forest views with no pathways to help promote a positive relation between mystery and preference? In this situation, will low visual access tend to translate into both high mystery and low preference? If so, the simple correlation between mystery and preference will be negative, and a positive correlation may not appear 
TABLE 1

Summary of Hypotheses

Hypothesis 1: Legibility and coherence will be independent positive predictors of preference.

Hypothesis 2: Visual access and landmarks will be independent positive predictors of legibility.

Hypothesis 3: The effects of either visual access or landmarks on preference will be mediated by the effect of legibility on preference.

Hypothesis 4: The correlation between preference and danger will be negative but not excessive in magnitude.

Hypothesis 5a: The pattern of relations for preference and danger with common predictors will differ from a polar-opposite pattern.

Hypothesis 5b: After controlling for visual access, mystery will have a positive relation with both preference and danger.

until visual access is statistically controlled. Such a pattern would bolster Herzog and Kutzli's suggestion that "strange things can happen to mystery when very low-access settings are involved" (p. 833). We were not sufficiently confident of this line of reasoning to propose the negative mysterypreference correlation as a formal hypothesis, but we certainly intended to check it out.

In summary, to test all of our formal hypotheses, we had independent groups of participants provide ratings on a single variable for a large sample of 70 within-forest settings containing no visible pathways. A summary of our hypotheses is presented in Table 1. Because they are directly involved in our hypotheses, we obtained ratings for the following variables: preference, danger, legibility, coherence, mystery, visual access, and landmarks. For the sake of completeness, we also obtained ratings for the remaining preferencematrix predictor: complexity. Finally, because Herzog and Kutzli (2002) presented some evidence that visual and locomotor access might be independent predictors of preference or danger, we also included a measure of locomotor access: movement ease.

\section{METHOD}

\section{PARTICIPANTS}

The sample of raters consisted of 400 undergraduate students (133 men, 267 women) at a university in the midwestern United States. Participants fulfilled a course requirement for introductory psychology by participating in the study. Twenty-seven sessions were run, with the number of participants per session ranging from 3 to 25 . 

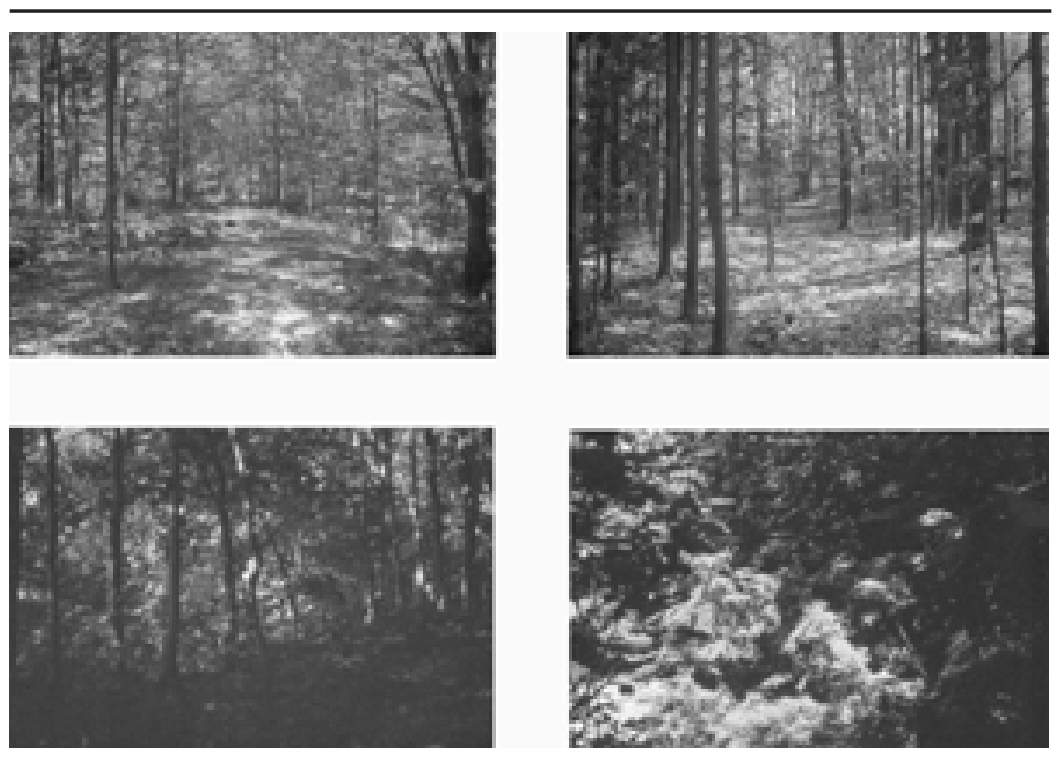

Figure 2: Settings Providing a Strong Contrast on Several of the Rated Variables NOTE: Settings in the top row tended to be high in preference, legibility, and visual access but low in danger and mystery. Settings in the bottom row tended to have the opposite pattern. Setting scores for the settings are in Table 2.

\section{STIMULI}

The settings consisted of 70 color slides of within-forest environments containing no visible pathways. It was, therefore, a purposive sample of settings. Additional criteria for scene selection included the following: a broad range of visual access in terms of depth of view and both the presence and absence of distinctive landmarks such as rock formations or unusually shaped trees. Figure 2 provides some imagery illustrating the range of values for visual access, legibility, and mystery. No settings contained people. All were photographed in spring, summer, or early fall. All slides were oriented horizontally.

\section{PROCEDURE}

All participants in each session rated each of the 70 settings on only one of the nine measured variables. All ratings used a 5-point scale ranging from A (very high [highest possible rating]) to $\mathrm{E}$ (not at all [lowest possible rating]). The letters A through $\mathrm{E}$ were later converted to the numbers 5 through 1 , 
respectively, for analysis. The target variable preference was defined as the following:

How much do you like the setting? This is your own personal degree of liking for the setting as a setting, NOT as a picture. You don't have to worry about whether you're right or wrong or whether you agree with anybody else.

The target variable danger was defined as the following: "How dangerous is this setting? How likely is it that you could be harmed in this setting?" For the four preference-matrix predictors, we used the standard definitions. Thus, coherence was defined as the following: "How well does the scene "hang together'? How easy is it to organize and structure the scene?" Complexity was defined as the following: "How much is going on in the scene? How much is there to look at? If the scene contains a lot of elements of different kinds, rate it high in complexity." Mystery was defined as the following: "How much does the setting promise more to be seen if you could walk deeper into it? Does the setting seem to invite you to enter more deeply into it and thereby learn more?" Legibility was defined as the following:

How easy would it be to find your way around in the setting? How easy would it be to figure out where you are at any given moment or to find your way back to any given point in the setting?

We use the Herzog and Leverich (2003) definition for the predictor landmarks: "To what extent does the setting contain distinctive or memorable objects or features that could serve as useful landmarks to help you find your way around in the setting?" For visual access and movement ease, we borrowed the definitions of Herzog and Kutzli (2002). Thus, visual access was defined as the following: "How easy is it to see into this setting? How well can you see all parts of this setting without having your view blocked or interfered with?" and movement ease was defined as the following: "How easy would it be to move within or through this setting?"

Sessions proceeded as follows: After explaining the task and obtaining informed consent, the first 10 slides were shown briefly ( 5 seconds per slide) without being rated to familiarize participants with the range of settings to be encountered. Then participants rated 74 slides, presented in two sets of 37 slides each, with a 2-minute rest between sets. The first and last slide within each set were fillers. The remaining 70 slides yielded the data for analysis. These slides were presented in one of two orders. The first order was used for the first 12 sessions, the second order for the last 15 sessions. We had to run more sessions with the second order because of a dearth of participant volun- 
teers during that period. Even with the extra sessions, we ended up with 256 participants using the first slide order (Sessions 1-12) and only 154 using the second slide order (Sessions 13-27). In the sessions using the first order, there were 4 sessions devoted to preference and 1 session devoted to each of the other eight variables. In the sessions using the second order, there were 6 sessions devoted to preference, 2 to movement ease, and 1 to each of the remaining seven variables. ${ }^{2}$ Aside from the constraints on the ordering of sessions just noted, the ordering of sessions was haphazard. One of the slide presentation orders was generated randomly, and the second presentation order was derived by interchanging the halves of the first order. Viewing time was 15 seconds per slide in all sessions. Final sample sizes were 170 for preference; 30 for coherence; 29 for complexity legibility, visual access, and movement ease; and 28 for danger, mystery, and landmarks.

\section{RESULTS}

Unless noted otherwise, all analyses were based on setting scores as raw scores. A setting score is the mean score for each setting based on all participants who completed one of the rating tasks. Thus, for each rated variable, every setting had a setting score, and settings typically were the units of analysis. Internal consistency reliability coefficients (Cronbach's alpha), based on settings as cases and participants as items, ranged from .88 for complexity to .98 for visual access.

To provide the reader with a feel for the variables, Table 2 contains the setting scores for each of the settings in Figure 2 on all nine rated variables. The means and standard deviations for the entire set of 70 settings on all nine rated variables are also included. The settings in each row of Figure 2 illustrate common combinations of relatively extreme values on several of the rated variables. As documented in Table 2, the settings in the top row of Figure 2 tended to be relatively high in preference, legibility, and visual access but low in danger and mystery. The settings in the bottom row of Figure 2 tended to have the opposite profile. Because these profiles were fairly common, we may anticipate that preference, legibility, and visual access were positively intercorrelated; so were danger and mystery, and the two sets of variables were negatively correlated with each other. Meanwhile, the overall means and standard deviations show that the ratings were fairly well centered along the rating scale, with a reasonable spread of scores for each variable. In general, the settings were seen as fairly low overall in danger and relatively high in legibility. 


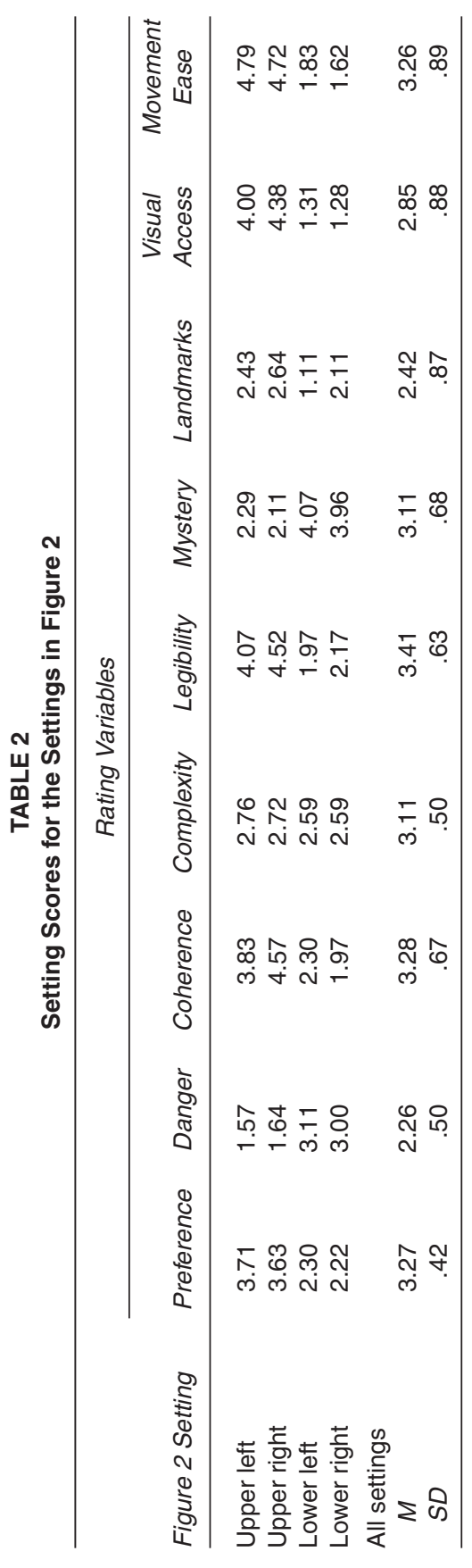


TABLE 3

Correlations Among All Rating Variables for All Settings $(N=70)$

\begin{tabular}{lllllllllll}
\hline Item & 1 & 2 & 3 & 4 & 5 & 6 & 7 & 8 & 9 \\
\hline
\end{tabular}

\begin{tabular}{|c|c|c|c|c|c|c|c|c|}
\hline 1. Preference & - & & & & & & & \\
\hline 2. Danger & $-.47^{\star \star}$ & - & & & & & & \\
\hline 3. Coherence & $.64^{* *}$ & $-.75^{\star \star}$ & - & & & & & \\
\hline 4. Complexity & $.33^{*}$ & .23 & .10 & - & & & & \\
\hline 5. Legibility & $.65^{\star \star}$ & $-.68^{\star \star}$ & $.71^{\star \star}$ & .16 & - & & & \\
\hline 6. Mystery & $-.39^{\star}$ & $.81^{\star *}$ & $-.70^{\star *}$ & -.04 & $-.68^{* *}$ & - & & \\
\hline 7. Landmarks & $.32^{\star}$ & .21 & .08 & $.54^{\star \star}$ & $.37^{\star}$ & .13 & - & \\
\hline 8. Visual access & $.55^{\star \star}$ & $-.86^{\star \star}$ & $.78^{\star \star}$ & .04 & $.77^{\star \star}$ & $-.92^{\star \star}$ & -.06 & - \\
\hline 9. Movement ease & $.42^{\star \star}$ & $-.88^{* *}$ & $.73^{\star \star}$ & $-.34^{*}$ & $.74^{\star *}$ & $-.70^{\star \star}$ & -.07 & $.80^{\star *}$ \\
\hline
\end{tabular}

${ }^{*} p<.01 .{ }^{* *} p<.001$.

\section{THE LEGIBILITY HYPOTHESES}

Indirect evidence pertinent to the legibility hypotheses (Hypotheses 1, 2, and 3) may be found in Table 3, which contains simple correlations among the nine rated variables based on all 70 settings. First, regarding Hypothesis 1 (that legibility and coherence would be independent positive predictors of preference), note that the simple correlations of legibility and coherence with preference were substantial and about equal (.64 and .65 , respectively). The correlation between the two predictors (.71) was not so great as to preclude significant partial relations between each predictor and preference. Second, regarding Hypothesis 2 (that visual access and landmarks would be independent positive predictors of legibility), note that the two predictors were positively correlated with legibility and uncorrelated with each other. Third, all of the simple correlations required by the mediation proposal of Hypothesis 3 were as expected. That is, visual access and landmarks were each correlated with both legibility and preference, and legibility and preference were correlated with each other.

The direct test of Hypothesis 1 was a multiple regression analysis with preference as the dependent variable and legibility and coherence as predictors. The results are presented in Table 4. As is evident, the hypothesis was supported. Legibility and coherence were independent positive predictors of preference.

The direct test of Hypothesis 2 was a multiple regression analysis with legibility as the dependent variable and visual access and landmarks as predictors. The results are presented in Table 5. Again, the hypothesis was supported. Visual access and landmarks were independent positive predictors of legibility. 
TABLE 4

Regression of Preference on Coherence and Legibility for All Settings ( $N=70)$

\begin{tabular}{llll}
\hline Predictor & $\mathrm{B}$ & Partial $\mathrm{r}$ & $\mathrm{p}$ \\
\hline Coherence & .22 & .32 & .006 \\
Legibility & .27 & .36 & .002 \\
\hline
\end{tabular}

NOTE: $B$ is the raw-score regression weight. Adjusted $R^{2}=.47, p<.001$.

TABLE 5

Regression of Legibility on Visual Access and Landmarks for All Settings $(N=70)$

\begin{tabular}{lllc}
\hline Predictor & B & Partial $r$ & $p$ \\
\hline Visual access & .58 & .86 & $<.001$ \\
Landmarks & .31 & .66 & $<.001$ \\
\hline
\end{tabular}

NOTE: $B$ is the raw-score regression weight. Adjusted $R^{2}=.77, p<.001$.

A direct test of the mediation hypothesis (Hypothesis 3) required a separate analysis for visual access and landmarks. In each case, we first had to verify that the proposed independent variable (visual access or landmarks) did not interact with the proposed mediator (legibility). If that hurdle was passed successfully, the mediation analysis was a multiple regression analysis with preference as the dependent variable and the proposed independent variable and the proposed mediator as predictors. Mediation would be indicated if the proposed mediator (legibility) was a significant predictor in the regression analysis, and the proposed independent variable (visual access or landmarks) was either greatly reduced or eliminated as a predictor. The interaction test was not significant for landmarks $(p=.759)$. The results of the mediation analysis are presented in Table 6 . As is evident, the results indicate full mediation in support of Hypothesis 3. That is, the mediator (legibility) remained a significant predictor in the multiple regression analysis, but the predictive power of landmarks was eliminated. In the case of visual access, its interaction with legibility was significant $(p=.007)$, precluding mediation analysis. The multiple regression equation for the interaction model was as follows:

$$
P^{\prime}=.18+.85 L+.81 A-.21 L A
$$

where $P$ ' is predicted preference, $L$ is legibility, and $A$ is visual access. By substituting extreme scale values ( 1 or 5 ) for either predictor in the equation, one can trace what happens to the relation between preference and the other predictor. What one finds is that for either predictor, the relation with 
preference is strongest and positive at the lowest scale value (1) for the other predictor and shrinks to near 0 (or perhaps slightly negative) at the highest scale value for the other predictor. In other words, the nature of the interaction is that either legibility or access works best at low values of the other predictor.

\section{THE PREFERENCE-DANGER HYPOTHESES}

The simple correlations in Table 3 bear directly on two of our formal hypotheses about preference and danger (and on a third, the mysterypreference correlation, that we could not bring ourselves to state formally). Regarding the formal hypotheses, note first that Hypothesis 4, which proposes a modest but significant negative correlation between preference and danger, was confirmed $(r=-.47)$. This means that it was at least possible for Hypothesis 5a to be confirmed. Hypothesis 5a proposed that the pattern of relations for preference and danger with common predictors would differ from a polar-opposite pattern. One way to test the hypothesis is to examine the pattern of correlations between the two target variables (preference and danger) and all seven of the predictor variables. Confirmation would require avoiding a polar-opposite pattern of predictor-target correlations for the two target variables. Table 3 shows that, generally, preference and danger had opposite-sign correlations with the predictor variables, but there were two exceptions. Both complexity and landmarks tended to be positively correlated with both target variables, although the correlations were significant $(p<.01)$ only for preference. This is very modest evidence in support of Hypothesis 5a. An alternative test of the hypothesis is described below. The unstated hypothesis (that there would be a negative simple correlation between mystery and preference) was confirmed $(r=-.39, p<.01)$.

An economical way to test Hypothesis 5 a further without having to worry about the large correlations among some of the predictor variables is to factor analyze the correlations among the predictors. We did so (principal axis factoring, varimax rotation) and the result was two factors that, after rotation, accounted for $55 \%$ and $19 \%$ of the variance in the data, respectively. Communalities ranged from .46 to .92 . The rotated factor loadings are presented in Table 7. The first factor seems to be about structure (coherence, legibility) and visibility (visual access, movement ease, mystery). If we think of structure as abetting visibility, then we can roughly summarize the factor as visibility. Note that in this data set, mystery detracts from visibility. The same point is emphasized by the very strong negative correlation (-.92) between mystery and visual access in Table 3 . The second factor is influenced primarily by landmarks and complexity. Both variables are concerned with the 
TABLE 6

Regression of Preference on Legibility and Landmarks for All Settings $(N=70)$

\begin{tabular}{lccr}
\hline Predictor & $\mathrm{B}$ & Partial $\mathrm{r}$ & $\mathrm{p}$ \\
\hline Legibility & .41 & .60 & $<.001$ \\
Landmarks & .04 & .11 & .379 \\
\hline
\end{tabular}

NOTE: $B$ is the raw-score regression weight. Adjusted $R^{2}=.41, p<.001$.

TABLE 7

Factor Loadings From the Factor Analysis of the Seven Predictor Variables

\begin{tabular}{|c|c|c|}
\hline \multirow[b]{2}{*}{ Variable } & \multicolumn{2}{|c|}{ Factors } \\
\hline & I & II \\
\hline Coherence & .83 & \\
\hline Complexity & & .68 \\
\hline Legibility & .86 & .36 \\
\hline Mystery & -.85 & \\
\hline Landmarks & & .82 \\
\hline Visual access & .96 & \\
\hline Movement ease & .87 & -.22 \\
\hline
\end{tabular}

NOTE: Only loadings greater than |.10| are shown. Factors I and II were interpreted as visibility and information, respectively.

amount of distinctive information in a setting, and thus the factor can be roughly summarized in terms of the amount of information to be processed. For informal description, we refer to the factors as visibility and information. We obtained factor scores using the regression approach for each of the two factors. The correlation between the factor scores for visibility and information was only .02 . Visibility was positively correlated with preference and negatively correlated with danger $(r=.59$ and -.88 , respectively, $p<.001$ in both cases). In contrast, information had modest positive correlations with both preference and danger $(r=.36, p<.01$, and $r=.26, p<.05$, respectively). The same trends appear even more clearly in multiple regression analyses with either target variable as the dependent variable and visibility and information as the predictors. The results in Table 8 show opposite-sign partial relations for visibility with the two target variables but positive partial relations $(p<.001)$ for information with both target variables. In sum, the pattern of relations for these factor-based predictors differed from a polar-opposite pattern for preference and danger, providing further support for Hypothesis $5 \mathrm{a}$.

Finally, Hypothesis $5 b$ (that mystery would have a positive relation with both preference and danger, after controlling for visual access) was tested by computing partial correlations between mystery and both target variables, 
TABLE 8

Regression of Preference and Danger on Visibility and Information ( $N=70$ Settings)

\begin{tabular}{lrrrrrrrr}
\hline & \multicolumn{3}{c}{ Preference } & & \multicolumn{3}{c}{ Danger } \\
\cline { 2 - 3 } \cline { 6 - 8 } Predictor & $\mathrm{B}$ & Partial $\mathrm{r}$ & $\mathrm{p}$ & & $\mathrm{B}$ & Partial $\mathrm{r}$ & $\mathrm{p}$ \\
\hline Visibility & .25 & .63 & $<.001$ & & -.44 & -.92 & $<.001$ \\
Information & .16 & .43 & $<.001$ & & .15 & .59 & $<.001$ \\
\hline
\end{tabular}

NOTE: $B$ is the raw-score regression weight. For preference, adjusted $R^{2}=.46, p<.001$; for danger, adjusted $R^{2}=.85, p<.001$.

preference and danger, with visual access partialed out. The partial correlations were $.34(p=.004)$ for preference and $.09(p=.447)$ for danger. Thus, Hypothesis $5 \mathrm{~b}$ was only partially supported. After controlling for visual access, mystery was positively related to preference but unrelated to danger.

\section{DISCUSSION}

As with all research, this study had potential limitations. Three that should be considered are participants, medium of presentation, and setting domain. First, our participant population was college students, and there is reason to suppose that the reactions of college students to environmental settings do not necessarily generalize to other age groups (Balling \& Falk, 1982; Herzog, Herbert, Kaplan, \& Crooks, 2000; Zube, Pitt, \& Evans, 1983). Second, we used color slides to present the settings. Although concerns about the generality of results from this medium of presentation have been raised (e.g., Heft \& Nasar, 2000; Hetherington, Daniel, \& Brown, 1993; Scott \& Canter, 1997), the validity of the medium for aggregate results and static visual attributes of environments is strongly supported (e.g., Hershberger \& Cass, 1973; Hull \& Stewart, 1992; Sommer, Summit, \& Clements, 1993; Trent, Neumann, \& Kvashny, 1987; Zube, Simcox, \& Law, 1987). Finally, there is good reason to believe that some of our findings apply narrowly to the domain of within-forest settings containing no pathways. Where this is likely, we will try to be clear about it.

Given these limitations, what can be concluded? First, the post hoc suggestions of Herzog and Leverich (2003), which led to the legibility hypotheses (Hypotheses 1, 2, and 3), were generally confirmed. Forest settings appear to be a good domain for showing empirically that legibility can predict preference independently from coherence (Hypothesis 1). Moreover, we were able to show that visual access and landmarks were each independent predictors of legibility in forest settings without pathways (Hypothesis 2). 
Finally, the positive relation between the presence of landmarks and preference was fully mediated by the effect of landmarks on legibility (Hypothesis 3). However, the same cannot be said for visual access. Although visual access contributed significantly to legibility, it also interacted with legibility in predicting preference. The nature of the interaction was that the relation between preference and either predictor was strongest and positive at low values of the other predictor. If we take the interaction seriously (and perhaps we should not until it is replicated), then a practical consequence would be that a planner could focus on either visual access or legibility, confident that if a high value on one of them could be achieved, the other one does not matter very much. In a more general sense, our results strongly confirm that in forest settings, legibility is important and that visual access and landmarks contribute to legibility.

Support for the hypotheses involving preference and danger was less consistent. Preference and danger had a modest negative correlation (Hypothesis 4 ), leaving plenty of room for different profiles of relations with predictors (Hypothesis 5a). It is important to be clear that Hypothesis 5a is supported when the profiles of relations with predictors for preference and danger depart from a polar-opposite pattern. Thus, the strongest support occurs when one can find predictors that have the same kind of relation (positive or negative) with both target variables. In the simple correlations, complexity and landmarks were the only predictors to show such a tendency, but only their positive correlations with preference were significant. These two predictors clustered together in our factor analysis of predictor intercorrelations to form a factor that we called information. As a composite predictor, information had a positive and significant, simple and partial relation with both preference and danger, supporting Hypothesis $5 \mathrm{a}$.

Hypothesis 5 b nominates mystery as a specific predictor that should have same-sign relations with both preference and danger. Such a pattern would also support Hypothesis 5a. Hypothesis $5 \mathrm{~b}$ adds the proviso that the samesign pattern of relations between mystery and both target variables will not manifest clearly until visual access is controlled statistically. The proviso was suggested by the partial correlations results of Herzog and Kutzli (2002). Unfortunately, we could not confirm this prediction. Partialing out visual access turned the correlation between mystery and preference from negative to positive, as expected, but it also eliminated the positive correlation between mystery and danger, contrary to prediction. If we abstract from several studies (Herzog \& Flynn-Smith, 2001; Herzog \& Kutzli, 2002; Herzog \& Miller, 1998), including this one, we can suggest that there is some tendency for mystery to be positively related to both preference and danger, after controlling for other predictors, but that the effect is highly context depen- 
dent. The implications of both Hypotheses $5 \mathrm{a}$ and $5 \mathrm{~b}$ are the same: Some predictors may contribute positively to both preference and danger. The challenge for planners and designers is to find ways to use the predictors to enhance preference without evoking danger. Useful suggestions can be found in Herzog and Kutzli (2002), Herzog and Leverich (2003), Herzog and Miller (1998), Kuo et al. (1998), and Nasar and Jones (1997).

The most challenging results of this study involve mystery. The unstated hypothesis in the introduction (that mystery might be negatively correlated with preference) was confirmed (Table 3: $r=-.39$ ). One might be tempted to make an adjustment for the number of correlations in Table 3 and thereby dismiss the mystery-preference correlation as not really significant. That is probably unwise because the mystery-preference correlation was almost certainly a byproduct of another correlation that cannot be dismissed. The correlation between mystery and visual access was -.92. For this setting domain, when asked to rate mystery using the traditional definition, the raters essentially defaulted to visual access, or perhaps antiaccess would be more appropriate. It was the low-access settings, such as those in the bottom row of Figure 2, that received the high mystery ratings. Because such settings were also low in preference, at least a modest negative correlation between mystery and preference was inevitable.

But why did this happen? Why were the low-access settings in the bottom row of Figure 2 rated higher in mystery than the high-access settings in the top row? One possibility is that there truly is a greater promise of more to be seen in the almost completely blocked views in the bottom row of Figure 2 than in the more open views in the top row. Some of the low-access settings contained a very dark foreground with at least a hint of brighter areas beyond (e.g., Figure 2, lower left). This is a classic pattern, indicative of mystery (R. Kaplan \& Kaplan, 1989, pp. 55-56). However, the hint of something beyond was meager because the foreground was almost completely blocked. The lack of visual access may have been the more potent (negative) influence on preference. On this interpretation, we seem to have found a special domain (forest settings without pathways) in which mystery has a genuinely negative relation with preference because of the way it relates to visual access. (Note that when visual access was controlled statistically, the preference-mystery relation reverted to the more commonly found positive correlation.) It may be the case that when pathways are present in forest settings, another classic pattern indicative of mystery - the bend in a pathway — coincides with a realignment of mystery and visual access such that both predictors are positively related to preference and to each other. This line of thought strongly suggests that a direct comparison of within-forest settings with and without pathways might be insightful. 
Finally, we offer a brief comment on movement ease. We included it because Herzog and Kutzli (2002) found that it predicted their target variables independently of other predictors concerned with visibility. In this study, that was not the case. Movement ease was part of the visibility cluster in the factor analysis. We suspect this discrepancy is a function of the set of predictor variables used in the two studies. The predictor variables in Herzog and Kutzli could all be seen as dealing with access, and thus a split between visual and locomotor access was possible. The predictors in this study dealt with both access and amount of information, leaving less leeway for a fine distinction between types of access to appear. As always, factors tend to depend on the types of variables included in a study.

In summary, one way to view our study is as an exploration of the rather specialized setting domain of within-forest settings without visible pathways. On that view, we think there are two major conclusions. First, legibility seems to be especially important in this domain, and it predicts preference independently from coherence. Moreover, landmarks and visual access contribute to legibility, and visual access probably also has predictive power of its own. Second, mystery, as traditionally defined, behaves oddly in this domain. Apparently, it defaults to lack of visual access because many of the settings highest in mystery are those with the foreground almost completely blocked by foliage and shadow but with just a hint of brighter areas in the distance. Such settings tend to be both disliked and perceived as potentially dangerous. Thus, in trying to understand preference or perceived danger in this domain, one might do better to ignore mystery and concentrate on visual access.

\section{NOTES}

1. If they do interact, one explores the nature of the interaction instead of doing mediation analysis.

2. The extra sessions for preference afforded us the option of factor analyzing the preference ratings. We did so (principal axis factoring, varimax rotation), and there were two major factors or categories of settings. These corresponded visually to the high and low visual access settings. The correlation between setting category and visual access was .89. Given this degree of redundancy between setting category and one of the rated predictors, we did not pursue further analyses involving setting category.

\section{REFERENCES}

Balling, J. D., \& Falk, J. H. (1982). Development of visual preference for natural environments. Environment and Behavior, 14, 5-28. 
Baron, R. M., \& Kenny, D. A. (1986). The moderator-mediator variable distinction in social psychological research: Conceptual, strategic, and statistical considerations. Journal of Personality and Social Psychology, 51, 1173-1182.

Evans, G. W., \& Lepore, S. J. (1997). Moderating and mediating processes in environmentbehavior research. In G. T. Moore \& R. W. Marans (Eds.), Advances in environment, behavior, and design (pp. 255-285). New York: Plenum.

Heft, H., \& Nasar, J. L. (2000). Evaluating environmental scenes using dynamic versus static displays. Environment and Behavior, 32, 301-322.

Hershberger, R. G., \& Cass, R. C. (1973). The adequacy of various media as representations of the designed environment. Man-Environment Systems, 3, 371-372.

Herzog, T. R., \& Flynn-Smith, J. A. (2001). Preference and perceived danger as a function of the perceived curvature, length, and width of urban alleys. Environment and Behavior, 33, 655668.

Herzog, T. R., Herbert, E. J., Kaplan, R., \& Crooks, C. L. (2000). Cultural and developmental comparisons of landscape perceptions and preferences. Environment and Behavior, 32, 323346.

Herzog, T. R., \& Kutzli, G. E. (2002). Preference and perceived danger in field/forest settings. Environment and Behavior, 34, 819-835.

Herzog, T. R., \& Leverich, O. L. (2003). Searching for legibility. Environment and Behavior, 35, 459-477.

Herzog, T. R., \& Miller, E. J. (1998). The role of mystery in perceived danger and environmental preference. Environment and Behavior, 30, 429-449.

Herzog, T. R., \& Smith, G. A. (1988). Danger, mystery, and environmental preference. Environment and Behavior, 20, 320-344.

Hetherington, J., Daniel, T. C., \& Brown, T. C. (1993). Is motion more important than it sounds? The medium of presentation in environment perception research. Journal of Environmental Psychology, 13, 283-291.

Hull, R. B., IV, \& Stewart, W. P. (1992). Validity of photo-based scenic beauty judgments. Journal of Environmental Psychology, 12, 101-114.

Kaplan, R., \& Kaplan, S. (1989). The experience of nature: A psychological perspective. New York: Cambridge University Press.

Kaplan, S., \& Kaplan, R. (1982). Cognition and environment: Functioning in an uncertain world. New York: Praeger.

Kuo, F. E., Bacaicoa, M., \& Sullivan, W. C. (1998). Transforming inner-city landscapes: Trees, sense of safety, and preference. Environment and Behavior, 30, 28-59.

Nasar, J. L., \& Jones, K. M. (1997). Landscapes of fear and stress. Environment and Behavior, 29, 291-323.

Scott, M. J., \& Canter, D. V. (1997). Picture or place? A multiple sorting study of landscape. Journal of Environmental Psychology, 17, 263-281.

Sommer, R., Summit, J., \& Clements, A. (1993). Slide ratings of street-tree attributes: Some methodological issues and answers. Landscape Journal, 12, 17-22.

Trent, R. B., Neumann, E., \& Kvashny, A. (1987). Presentation mode and question format artifacts in visual assessment research. Landscape and Urban Planning, 14, 225-235.

Zube, E. H., Pitt, D. G., \& Evans, G. W. (1983). A lifespan developmental study of landscape assessment. Journal of Environmental Psychology, 3, 115-128.

Zube, E. H., Simcox, D. E., \& Law, C. S. (1987). Perceptual landscape simulations: History and prospect. Landscape Journal, 6, 62-80. 